\title{
Sustained oscillations in the MAP kinase cascade
}

\author{
Juliette Hell $^{1}$ and Alan D. Rendall ${ }^{2}$ \\ ${ }^{1}$ Freie Universität Berlin, jhell@zedat.fu-berlin.de. \\ ${ }^{2}$ Johannes Gutenberg-Universität Mainz, rendall@uni-mainz.de.
}

\begin{abstract}
The MAP kinase cascade is a network of enzymatic reactions arranged in layers. In each layer occurs a multiple futile cycle of phosphorylations. The fully phosphorylated substrate then serves as an enzyme for the layer below. This papers focusses on the existence of parameters for which Hopf bifurcations occur and generate periodic orbits. Furthermore it is explained how geometric singular perturbation theory allows to generalize results from simple models to more complex ones.
\end{abstract}

\section{Introduction}

The MAP kinase cascade (mitogen-activated protein kinase cascade) is a pattern of chemical reactions encountered frequently in cell biology. The actual substances involved in the reactions vary from one example to another but certain features are always present. There are three proteins, generically denoted by MAPK, MAPKK and MAPKKK. For brevity we mostly use the notations $K, K K$ and $K K K$ for these. A phosphate group can be attached to $K K K$ at a particular site and this causes it to become activated. It then catalyses the addition of phosphate groups to $K K$ at two sites. An enzyme which catalyses a phosphorylation in this way is called a kinase and MAPKKK stands for MAPKK kinase. Similarly when $K K$ has been phosphorylated at both sites it becomes activated and catalyses the addition of phosphate groups to $K$ at two sites. There are also other enzymes (phosphatases) which remove phosphate groups from each of the three proteins. We refer to one of the proteins $K, K K$ or $K K K$ together with its phosphorylated forms and the reactions interconverting these forms as a layer of the cascade. We think of the layers corresponding to $K K K, K K$ and $K$ as being arranged from top to bottom and refer to them as the first, second and third layers. Since substances in one layer directly influence the reactions in the next layer information flows from the top to the bottom in this picture and that is the reason it is called a 
cascade. The MAPK cascade can be represented in the following way:

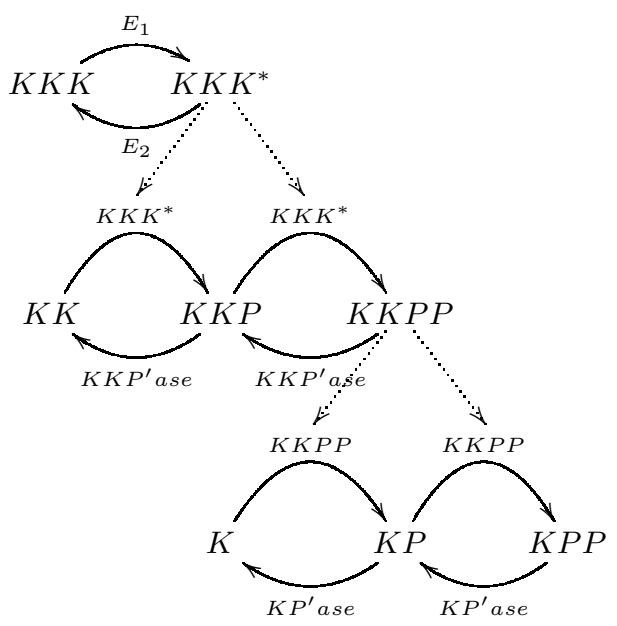

In this diagram, an enzymatic reaction $X \stackrel{E}{\longrightarrow} Y$ represents the chemical reactions $X+E \rightleftarrows X \cdot E \rightarrow Y+E$, where $X \cdot E$ is a complex of substrate $X$ and enzyme $E$. In fact information can flow upwards through the cascade in a more indirect sense and this is described in more detail below. This backward flow has an essential influence on the dynamics of the system.

If each of the reactions is modelled by a standard irreversible MichaelisMenten scheme (see for instance [23]) composed of three elementary reactions with substrate, enzyme and substrate-enzyme complex and the elementary reactions are given mass action kinetics a system of ordinary differential equations arises as a model for the MAPK cascade. This will be referred to as the Huang-Ferrell model since it was introduced by those authors in [17. In that paper they studied mathematical properties of these equations and also compared solutions of the equations with the results of the experiments they had done on a MAPK cascade in extracts of oocytes (immature egg cells) of the frog Xenopus. The full system consists of 22 equations (for 8 substrates, 4 enzymes which do not occur as substrates and 10 complexes) and depends on 30 parameters (reaction constants). The procedure in [17 was to fix all but one of the parameters and numerically determine stationary solutions of the system for different values of the remaining parameter. Then the value of the concentration of one of the substances at the stationary solution was studied as a function of the chosen parameter. This function exhibits the property of ultrasensitivity where the output is a sigmoid function of the input.

The possibility of carrying out this procedure is dependent on the fact that there exist a stationary solution for each value of the parameters. Moreover the results will only be unambiguous if there is only one such solution for fixed values of the reaction constants and the total amounts of the three proteins and the four other enzymes. This issue is not addressed in [17. That the answer to the uniqueness question is not obvious is made clear by the results of [22]. That paper was concerned with a system 
which can be thought of as a single layer of the MAPK cascade with two phosphorylation steps. The ODE system in this case is known as the dual futile cycle [33. The numerical and heuristic work in [22] indicated that this system exhibits bistability, i.e. that there are parameter values for which there exists more than one stable stationary solution. A rigorous proof that this is the case was given in [16].

A priori it is not ruled out that solutions of the Huang-Ferrell model might exhibit more complicated long-time behaviour than just convergence to a stationary solution. In fact, numerical and heuristic work in 26 indicates that periodic solutions exist. There is also evidence suggesting that chaotic behaviour may occur 35. According to the investigations of 26] periodic solutions already occur in the system corresponding to the first two layers of the MAPK cascade. We refer to this system as the truncated Huang-Ferrell model. In what follows we will prove that for both the truncated and full Huang-Ferrell models there exist parameters for which there are periodic solutions. This means that the protein concentrations being modelled undergo sustained oscillations.

Following the terminology of [14] we refer to the Huang-Ferrell model or an analogous system for a subset of the layers of the cascade as the MM-MA system (for Michaelis-Menten via mass action). Under suitable circumstances it is possible to derive a smaller system, the MM system (for Michaelis-Menten), via a quasistationary approximation. This is relatively simple to do for the dual futile cycle and the dynamics of the MM system in that case was studied in [24]. In that paper the authors found that bistability is already present in the MM system. The method used in 16, which will be generalized here, is to first prove bistability for the MM system and then use the fact that the MM system is a limit of the MM-MA system in a suitable sense to obtain the corresponding result for the MM-MA system. For this we used that the stationary solutions are hyperbolic. The technique applied to treat the singular limit is geometric singular perturbation theory (GSPT) 9]. In [16] an MM limit was defined for the truncated Huang-Ferrell model. The definition was inspired by ideas in 30 and 32. In these papers it was pointed out that the phenomenenon of sequestration can lead to a flow of information from layers further down in the cascade to higher levels. For instance, if a lot of $K K K^{*}$, the activated form of $K K K$, is bound to its substrates $K K$ and the phosphorylated form $K K P$ then not much of it is available as a substrate for the phosphatase which would otherwise convert it back to the inactivated form $K K K$. Related ideas are discussed in [6], where this effect is called retroactivity. It was mentioned in [16 that if it could be proved that this MM system admits a hyperbolic periodic solution then the truncated Huang-Ferrell system would also admit a periodic solution. It turned out to be difficult to obtain a proof of hyperbolicity and thus we will use a slightly different strategy in what follows. The basic idea is nevertheless still to first prove a result for the MM system and then lift some of the structure found to the MM-MA system.

The paper is organized as follows. Section 2 introduces some basic notation and terminology and Section 3 proceeds to show that the MM system derived from the truncated Huang-Ferrell model admits a Hopf bifurcation for certain values of the parameters. It is then shown that 
it can be concluded that the MM-MA system also admits a Hopf bifurcation. It follows from the basic theorem of Hopf (cf. [15]) that there are parameters for which the MM-MA system admits periodic solutions. These results are summed up in Theorem 1. Section 4 shows that the bistability in the dual futile cycle implies the presence of bistability in the truncated Huang-Ferrell model. In Section 5 the arguments of Section 3 are extended so as to prove Theorem 2 which asserts the existence of periodic solutions for the full Huang-Ferrell model. In particular this involves the use of an MM system for the full MAPK cascade. Section 6 presents results on some variants of the models coming from the MAPK cascade. For a cascade consisting of two single phosphorylation loops it is proved that all solutions of the MM system converge to the same stationary solution. In particular there are no sustained oscillations for that model. For a cascade consisting of a layer with two phosphorylations above a layer with one phosphorylation it is not clear whether sustained oscillations can occur. It is shown how this configuration can arise by modelling a system considered in 25], thus motivating further study of this question. The final section indicates some directions in which the results of this paper might be extended. Some information on geometric singular perturbation theory is collected in an appendix.

\section{The basic equations}

In what follows we essentially adopt the notations of Huang and Ferrell [17. for the full cascade and then specialize them to the truncated system.

The unknowns in the system are the concentrations

$$
\begin{aligned}
& {[K K K],\left[K K K^{*}\right],\left[E_{1}\right],\left[E_{2}\right],\left[K K K \cdot E_{1}\right],\left[K K K^{*} \cdot E_{2}\right],} \\
& {[K K],[K K P],[K K P P],\left[K K P^{\prime} \text { ase }\right],} \\
& {\left[K K \cdot K K K^{*}\right],\left[K K P \cdot K K K^{*}\right],\left[K K P \cdot K K P^{\prime} \text { ase }\right],\left[K K P P \cdot K K P^{\prime} \text { ase }\right],} \\
& {[K],[K P],[K P P],\left[K P^{\prime} a s e\right]} \\
& {[K \cdot K K P P],[K P \cdot K K P P],\left[K P \cdot K P^{\prime} \text { ase }\right],\left[K P P \cdot K P^{\prime} \text { ase }\right] .}
\end{aligned}
$$

The square brackets around a symbol indicate the concentration of the substance denoted by that symbol. Here $E_{1}$ and $E_{2}$ are the kinase and the phosphatase in the first layer while $K K P^{\prime}$ ase and $K P^{\prime}$ ase are the phosphatases in the second and third layers. $K K K^{*}$ is the activated (i.e. phosphorylated) form of $K K K$. A $P$ occurring in the name of a protein indicates a phosphate group. For instance $K K P P$ is the doubly phosphorylated form of $K K$. Finally, the substrate-enzyme complexes are denoted by the symbols for the two substances separated by a centred dot. The full set of 22 evolution equations for these quantities will not be reproduced here. They can be found in [17. More precisely, 18 of them can be found there and the other four, those for the concentrations of the free enzymes, are easily derived from those. There are seven conserved quantities, three corresponding to the total amounts of the three substrate proteins in all phosphorylation states and four corresponding to the total amounts of the four enzymes. These conservation laws can be substituted into the evolution equations so as to reduce their number. In [17 the four 
conserved quantities associated to the enzymes are used in this way while the others are not. Thus 18 evolution equations remain. The reaction constants for the elementary reactions in the Michaelis-Menten description are denoted by $\tilde{a}_{i}$ for the formation of the complex, $k_{i}$ for the liberation of product and $\tilde{d}_{i}$ for the release of the substrate from the complex. These notations are as in [17 except that $a_{i}$ and $d_{i}$ have been replaced by $\tilde{a}_{i}$ and $\tilde{d}_{i}$ since $a_{i}$ and $d_{i}$ will be used for a different purpose later. All reaction constants are assumed positive and since the unknowns in the equations are concentrations the solutions of interest are those which are positive, i.e. all their components are positive.

The truncated Huang-Ferrell model is obtained by setting the concentrations of all the unknowns in the full model involving the substance $K$ and that of the enzyme $K P^{\prime}$ ase (i.e. the quantities in the last two lines of (2) to zero and discarding the evolution equations for those quantities. For the rest of this section we concentrate on the truncated model. This serves, in particular, to introduce some of the main techniques of the paper in a context simpler than that of the full cascade. The results for the truncated cascade also constitute a central component of the proof of the theorems for the full cascade. As explained in [16] the passage to the limiting MM system can be carried out by scaling the variables in a certain way with powers of a parameter $\epsilon$ and then letting $\epsilon$ tend to zero. First it is important to introduce a new variable to replace $\left[K K K^{*}\right]$. This is

$$
\overline{K K K}=\left[K K K^{*}\right]+\left[K K \cdot K K K^{*}\right]+\left[K K P \cdot K K K^{*}\right] .
$$

When the Huang-Ferrell model is written in terms of $\overline{K K K}$ the equations of the truncated model are a subset of the equations for the full model. The scaling is done as follows. The quantities in the evolution equations are replaced by new quantities defined in the following way. The concentrations of compounds not containing $K K K$ or the enzymes $E_{i}$ or $K K P^{\prime}$ ase are not rescaled. These are the first three quantities in the second line of (2). For the concentrations of compounds which contain $K K K$ or $K K P^{\prime}$ ase but not the $E_{i}$ the new quantity is $\epsilon^{-1}$ times the old one. These are the first two quantities in the first line, the last quantity in the second line and all the quantities in the third line of (2). For the quantities involving $E_{1}$ and $E_{2}$ the new quantity is $\epsilon^{-2}$ times the old one. These are the last four quantities in the first line of (2). The reaction constants $\tilde{a}_{1}$ and $\tilde{a}_{2}$ are multiplied by $\epsilon$ to get new quantities. All the other reaction constants are left unchanged. In addition a new time coordinate is introduced as $\epsilon$ times the old one. To avoid complicating the notation the rescaled quantities will be denoted in the same way as the original ones. There results the following system, which was given in a different notation in [16.

$$
\begin{aligned}
& \frac{d}{d t}(\overline{K K K})=-\tilde{a}_{2}\left[K K K^{*}\right]\left[E_{2}\right]+\tilde{d}_{2}\left[K K K^{*} \cdot E_{2}\right]+k_{1}\left[K K K \cdot E_{1}\right] \\
& \frac{d}{d t}[K K]=-\tilde{a}_{3}[K K]\left[K K K^{*}\right]+\tilde{d}_{3}\left[K K \cdot K K K^{*}\right]+k_{4}\left[K K P \cdot K K P^{\prime} \text { ase }\right] \\
& \frac{d}{d t}[K K P P]=-\tilde{a}_{6}[K K P P]\left[K K P^{\prime} \text { ase }\right]+\tilde{d}_{6}\left[K K P P \cdot K K P^{\prime} \text { ase }\right]+k_{5}[K K P \cdot K K K(\phi)
\end{aligned}
$$




$$
\begin{aligned}
& \epsilon \frac{d\left[K K K \cdot E_{1}\right]}{d t}=\tilde{a}_{1}[K K K]\left[E_{1}\right]-\left(\tilde{d}_{1}+k_{1}\right)\left[K K K \cdot E_{1}\right] \\
& \epsilon \frac{d\left[K K K^{*} \cdot E_{2}\right]}{d t}=\tilde{a}_{2}\left[K K K^{*}\right]\left[E_{2}\right]-\left(\tilde{d}_{2}+k_{2}\right)\left[K K K^{*} \cdot E_{2}\right] \\
& \epsilon \frac{d\left[K K \cdot K K K^{*}\right]}{d t}=\tilde{a}_{3}[K K]\left[K K K^{*}\right]-\left(\tilde{d}_{3}+k_{3}\right)\left[K K \cdot K K K^{*}\right] \\
& \epsilon \frac{d\left[K K P \cdot K K K^{*}\right]}{d t}=\tilde{a}_{5}[K K P]\left[K K K^{*}\right]-\left(\tilde{d}_{5}+k_{5}\right)\left[K K P \cdot K K K^{*}\right], \\
& \epsilon \frac{d\left[K K P \cdot K K P^{\prime} \text { ase }\right]}{d t}=\tilde{a}_{4}[K K P]\left[K K P^{\prime} \text { ase }\right]-\left(\tilde{d}_{4}+k_{4}\right)\left[K K P \cdot K K P^{\prime} \text { ase }\right], \\
& \epsilon \frac{d\left[K K P P \cdot K K P^{\prime} a s e\right]}{d t}=\tilde{a}_{6}[K K P P]\left[K K P^{\prime} \text { ase }\right]-\left(\tilde{d}_{6}+k_{6}\right)\left[K K P P \cdot K K P^{\prime} \text { ase }\right] .
\end{aligned}
$$

These equations correspond to a subset of the equations given in [17. To get a closed system we must express some of the quantities on the right hand side of the equations in terms of those on the left hand side using the definition of $\overline{K K K}$ and the conservation laws. The necessary equations are

$$
\begin{aligned}
& {\left[K K K^{*}\right]=\overline{K K K}-\left[K K \cdot K K K^{*}\right]-\left[K K P \cdot K K K^{*}\right]} \\
& {[K K K]=K K K_{\mathrm{tot}}-\overline{K K K}+O(\epsilon)} \\
& {[K K P]=K K_{\mathrm{tot}}-[K K]-[K K P P]+O(\epsilon)} \\
& {\left[E_{1}\right]=E_{1, \mathrm{tot}}-\left[K K K \cdot E_{1}\right]} \\
& {\left[E_{2}\right]=E_{2, \mathrm{tot}}-\left[K K K^{*} \cdot E_{2}\right]} \\
& \left.\left[K K P^{\prime} \text { ase }\right]=\left(K K P^{\prime} \text { ase }\right)_{\mathrm{tot}}-\left[K K P \cdot K K P^{\prime} \text { ase }\right]-\left[K K P P \cdot K K P^{\prime} \text { (18 }\right]\right)
\end{aligned}
$$

This system of equations extends smoothly to $\epsilon=0$. The terms written as $O(\epsilon)$ are sums of concentrations of complexes. They are not written explicitly since they vanish for $\epsilon=0$ and thus make no contribution to the limiting equations. When $\epsilon=0$ the equations (7)-(12) become algebraic equations. Substituting these into the other evolution equations gives a set of three evolution equations, the MM system, which will now be computed. First the concentrations of the complexes can be expressed in terms of those of the substrates and the free enzymes.

$$
\begin{aligned}
& {\left[K K K \cdot E_{1}\right]=\frac{\tilde{a}_{1}[K K K]\left[E_{1}\right]}{\tilde{d}_{1}+k_{1}},} \\
& {\left[K K K^{*} \cdot E_{2}\right]=\frac{\tilde{a}_{2}\left[K K K^{*}\right]\left[E_{2}\right]}{\tilde{d}_{2}+k_{2}},} \\
& {\left[K K \cdot K K K^{*}\right]=\frac{\tilde{a}_{3}[K K]\left[K K K^{*}\right]}{\tilde{d}_{3}+k_{3}},} \\
& {\left[K K P \cdot K K K^{*}\right]=\frac{\tilde{a}_{5}[K K P]\left[K K K^{*}\right]}{\tilde{d}_{5}+k_{5}},} \\
& {\left[K K P \cdot K K P^{\prime} \text { ase }\right]=\frac{\tilde{a}_{4}[K K P]\left[K K P^{\prime} \text { ase }\right]}{\tilde{d}_{4}+k_{4}},} \\
& {\left[K K P P \cdot K K P^{\prime} \text { ase }\right]=\frac{\tilde{a}_{6}[K K P P]\left[K K P^{\prime} \text { ase }\right]}{\tilde{d}_{6}+k_{6}} .}
\end{aligned}
$$

It is convenient to introduce the Michaelis constants $K_{m, i}=\frac{\tilde{d}_{i}+k_{i}}{\tilde{a}_{i}}$. Now the concentrations of the free enzymes can be expressed in terms of the 
total concentrations of the enzymes. Note that

$$
\begin{aligned}
& E_{1, \text { tot }}=\left[E_{1}\right]\left(1+K_{m, 1}^{-1}[K K K]\right) \\
& E_{2, \text { tot }}=\left[E_{2}\right]\left(1+K_{m, 2}^{-1}\left[K K K^{*}\right]\right) \\
& \overline{K K K}=\left[K K K^{*}\right]\left(1+K_{m, 3}^{-1}[K K]+K_{m, 5}^{-1}[K K P]\right) \\
& \left(K K P^{\prime} \text { ase }\right)_{\text {tot }}=\left[K K P^{\prime} \text { ase }\right]\left(1+K_{m, 4}^{-1}[K K P]+K_{m, 6}^{-1}[K K P P] 28\right.
\end{aligned}
$$

Substituting this into the equations

$$
\begin{aligned}
& \frac{d}{d t}(\overline{K K K})=-\frac{k_{2}}{K_{m, 2}}\left[K K K^{*}\right]\left[E_{2}\right]+\frac{k_{1}}{K_{m, 1}}[K K K]\left[E_{1}\right] \\
& \frac{d}{d t}[K K]=-\frac{k_{3}}{K_{m, 3}}[K K]\left[K K K^{*}\right]+\frac{k_{4}}{K_{m, 4}}[K K P]\left[K K P^{\prime} \text { ase }\right] \\
& \frac{d}{d t}[K K P P]=-\frac{k_{6}}{K_{m, 6}}[K K P P]\left[K K P^{\prime} \text { ase }\right]+\frac{k_{5}}{K_{m, 5}}[K K P]\left[K K\left(33^{*} 1\right]\right)
\end{aligned}
$$

gives

$$
\begin{aligned}
& \frac{d}{d t}(\overline{K K K})=\frac{k_{1} K_{m, 1}^{-1} E_{1, \mathrm{tot}}[K K K]}{1+K_{m, 1}^{-1}[K K K]} \\
& -\frac{k_{2} K_{m, 2}^{-1} E_{2, \mathrm{tot}} \overline{K K K}}{1+K_{m, 2}^{-1} \overline{K K K}+K_{m, 3}^{-1}[K K]+K_{m, 5}^{-1}[K K P]}, \\
& \frac{d}{d t}[K K]=-\frac{k_{3} K_{m, 3}^{-1} \overline{K K K}[K K]}{1+K_{m, 3}^{-1}[K K]+K_{m, 5}^{-1}[K K P]} \\
& +\frac{k_{4} K_{m, 4}^{-1}\left(K K P^{\prime} a s e\right)_{\mathrm{tot}}[K K P]}{1+K_{m, 4}^{-1}[K K P]+K_{m, 6}^{-1}[K K P P]}, \\
& \frac{d}{d t}[K K P P]=+\frac{k_{5} K_{m, 5}^{-1} \overline{K K K}[K K P]}{1+K_{m, 3}^{-1}[K K]+K_{m, 5}^{-1}[K K P]} \\
& -\frac{k_{6} K_{m, 6}^{-1}\left(K K P^{\prime} a s e\right)_{\mathrm{tot}}[K K P P]}{1+K_{m, 4}^{-1}[K K P]+K_{m, 6}^{-1}[K K P P]} .
\end{aligned}
$$

Equations (32)-(34) are the Michaelis-Menten system. For convenience we introduce the notations $a_{i}=k_{i+2} K_{m, i+2}^{-1}$ for $i=1,3, a_{i}=k_{i+2} K_{m, i+2}^{-1}\left(K K P^{\prime} \text { ase }\right)_{\text {tot }}$ for $i=2,4, c_{i}=k_{i} K_{m, i}^{-1} E_{i, \text { tot }}$ for $i=1,2$ and $d_{i}=K_{m, i}^{-1}$ for $i=1,2$. Furthermore we replace $[K K P],[K K K]$ by their expressions in the chosen phase variables $\overline{K K K},[K K],[K K P P]$ and the parameters of the system.

Assume that the coefficients $K_{m, i}^{-1}$ are equal to a common quantity $b_{1}$ for $3 \leq i \leq 6$. For any choice of the parameters $a_{i}, b_{1}, c_{i}$ and $d_{i}$ there exists a choice of the parameters and total quantities of the enzymes for the MM-MA system which gives rise to them. This choice can be made so as to depend smoothly on $\left(a_{i}, b_{1}, c_{i}, d_{i}\right)$.

In this notation, the Michaelis-Menten system for the truncated cascade is the following:

$$
\frac{d}{d t}(\overline{K K K})=\frac{c_{1}\left(K K K_{\mathrm{tot}}-\overline{K K K}\right)}{1+d_{1}\left(K K K_{\mathrm{tot}}-\overline{K K K}\right)}
$$




$$
\begin{aligned}
& -\frac{c_{2} \overline{K K K}}{1+d_{2} \overline{K K K}+b_{1}\left(K K_{\mathrm{tot}}-[K K P P]\right.}, \\
& \frac{d}{d t}[K K]=-\frac{a_{1} \overline{K K K}[K K]}{1+b_{1}\left(K K_{\mathrm{tot}}-[K K P P]\right)} \\
& +\frac{a_{2}\left(K K_{\mathrm{tot}}-[K K]-[K K P P]\right)}{1+b_{1}\left(K K_{\mathrm{tot}}-[K K]\right)}, \\
& \frac{d}{d t}[K K P P]=+\frac{a_{3} \overline{K K K}\left(K K_{\mathrm{tot}}-[K K]-[K K P P]\right)}{1+b_{1}\left(K K_{\mathrm{tot}}-[K K P P]\right.} \\
& -\frac{a_{4}[K K P P]}{1+b_{1}\left(K K_{\mathrm{tot}}-[K K P P]\right)} .
\end{aligned}
$$

\section{Analysis of the truncated system}

The aim of this section is to prove that there are values of the parameters and the total amounts of substrates and enzymes in the truncated HuangFerrell system for which there exist periodic solutions. The first step is to determine explicit stationary solutions of the system (35)-(37) for certain values of the total amounts. The existence of periodic solutions of the MM system is obtained by showing that a Hopf bifurcation occurs at these stationary solutions. It is then shown that there is also a Hopf bifurcation, and hence periodic solutions, for the full truncated Huang-Ferrell system. Suppose that all parameters $a_{i}, b_{1}, c_{2}$ and $d_{i}$ have been fixed. Consider stationary solutions of (35)-(37) which satisfy $[K K]=[K K P P]$. Then $a_{1} \overline{K K K}[K K]=a_{2}[K K P]$ and $a_{3} \overline{K K K}[K K P]=a_{4}[K K P P]$ as a consequence of (36) and (37). In particular the product of these two equations gives $\overline{K K K}^{2}=\frac{a_{2} a_{4}}{a_{1} a_{3}}$ and so $\overline{K K K}$ is determined. Next it is possible to determine $[K K]$ and $[K K P]$ as functions of $K K_{\text {tot }}$ by means of the formulae

$$
[K K]=\frac{K K_{\mathrm{tot}}}{2+q_{1}}, \quad[K K P]=\frac{q_{1} K K_{\mathrm{tot}}}{2+q_{1}},
$$

where $q_{1}=\sqrt{\frac{a_{1} a_{4}}{a_{2} a_{3}}}$. The first equation (35) has not been used yet and produces a constraint on the parameters. This is the price to pay for simplifying some computations by having an equilibrium whose two last coordinates are identical. Set $Q=\frac{c_{2} \overline{K K K}}{1+d_{2} \overline{K K K}+b_{1}\left(K K_{\mathrm{tot}}-[K K]\right)}$. It follows from the equation $d \overline{K K K} / d t=0$ that $\frac{c_{1}\left(K K K_{\text {tot }}-\overline{K K K}\right)}{1+d_{1}\left(K K K_{\text {tot }}-\overline{K K K}\right)}=Q$. This is equivalent to $K K K_{\text {tot }}=\overline{K K K}+\frac{Q}{c_{1}-d_{1} Q}$ provided the denominator in the last term is positive. Choose $c_{1}$ so that $\frac{c_{2} d_{1}}{c_{1} d_{2}}<1$. Then the denominator is positive and this expression can be used to define $K K K_{\text {tot }}$. Once this has been done all the conditions for stationary solutions are satisfied. Let 
us summarize this information:

$$
\begin{aligned}
& \text { coordinates of the equilibrium } \overline{K K K}=\sqrt{\frac{a_{2} a_{4}}{a_{1} a_{3}}} \\
& {[K K]=[K K P P]=\frac{K K_{\mathrm{tot}}}{2+\sqrt{\frac{\cos _{1} a_{4}}{a_{2} a_{3}}}}} \\
& \text { constraints on the parameters } \frac{c_{2} d_{1}}{c_{1} d_{2}}<1 \\
& \begin{array}{l}
\frac{c_{2} d_{1}}{c_{1} d_{2}}<1 \\
K K K_{\text {tot }}=\sqrt{\frac{a_{2} a_{4}}{a_{1} a_{3}}}+\frac{Q}{c_{1}-d_{1} Q},
\end{array} \\
& \text { where } Q=\frac{c_{2} \overline{K K K}}{1+d_{2} \overline{K K K}+b_{1}\left(K K_{\mathrm{tot}}-[K K]\right)}
\end{aligned}
$$

Next the linearization of (35)-(37) will be considered. It turns out that the signs of all entries in the derivative of the right hand side of the system are independent of the values of the concentrations and can be determined. One is zero, one is positive and all the rest are negative. The characteristic polynomial of the linearization is the determinant of a matrix of the form

$$
\left[\begin{array}{ccc}
\lambda+a & 0 & c \\
d & \lambda+e & f \\
-g & h & \lambda+i
\end{array}\right]
$$

where all parameters are positive. Note that $-e,-f,-h$ and $-i$ only differ from the elements of the matrix (46) of [16] by the multiplicative factor $N^{-2}$ and the fact that $a_{1}$ and $a_{3}$ are replaced by $a_{1} \overline{K K K}$ and $a_{3} \overline{K K K}$, respectively. As in that case the parameters $a_{1} \overline{K K K}$ and $a_{4}$ can be eliminated. Let $M_{2}$ be the submatrix of the matrix (40) with $\lambda=0$ consisting of the second and third rows and columns. The determinant of the linearization is given by

$$
-\left(a\left|M_{2}\right|+c(d h+e g)\right) .
$$

Since we are seeking parameters for which a Hopf bifurcation producing stable periodic orbits takes place, the three eigenvalues of the linearization at the equilibrium (39) should be two imaginary eigenvalues $\pm i \omega_{0}, \omega_{0} \neq 0$, and a third real negative eigenvalue. In particular the determinant of the linearization and its trace should be both strictly negative. The sign of $\left|M_{2}\right|$ is unclear, but the linearization has a negative determinant if $\left|M_{2}\right|$ is small enough. Define $u=b_{1} K K_{\text {tot }}$ and $v=b_{1}[K K]$. Then the determinant of $M_{2}$ is given, up to a positive multiplicative constant, by

$$
-(u-3 v+1)(u-2 v) u(u-v+1)
$$

In fact all factors except the first are positive for all allowed values of the variables. Thus the determinant is negative iff $u-3 v+1>0$. This is equivalent to the condition $e i-f h<0$. In terms of the parameters of the reduced system $\left.u-3 v+1=\left(\frac{q_{1}-1}{q_{1}+2} b_{1} K K_{\text {tot }}+1\right)\right)$. It follows that provided $q_{1}<1$ the value of $K K_{\text {tot }}$ can be varied so that the determinant of $M_{2}$ passes from being positive to being negative.

Now consider the determinant of the matrix in the linearized system. It is equal to $-a(e i-f h)-c(d h+e g)$. The second term is automatically negative and so if $e i-f h>0$ the determinant of the full matrix is negative. On the other hand if $e i-f h<0$ the sign of the determinant is 
not clear. The trace is negative for all parameter values. In a deformation as above the determinant of the three-dimensional matrix is negative when the determinant of $M_{2}$ becomes zero. It stays negative at least in a small neighbourhood of that point. Thus there are parameters for which both the determinant of $M_{2}$ and the determinant of the three-dimensional matrix are negative. The determinant of (40) is

$$
\lambda^{3}+(a+e+i) \lambda^{2}+(a e+a i+c g+e i-f h) \lambda+(a e i-a f h+c d h+c g e) .
$$

Call it $p_{3}(\lambda)$. The eigenvalue conditions for a Hopf bifurcation are that two eigenvalues are purely imaginary and non-zero and that the third is non-zero. If this is true the trace is given by the real eigenvalue and thus determines its sign. In the example here we see that at a Hopf bifurcation the real eigenvalue must be negative. The determinant is then also negative. Introduce the notation $p_{3}(\lambda)=\lambda^{3}+A_{2} \lambda^{2}+A_{1} \lambda+A_{0}$. In a deformation as above all $A_{i}$ are positive near the point where $e i-f h=0$. The Routh-Hurwitz theorem [1] implies that all roots of this polynomial have negative real parts if and only if $A_{i}>0$ for all $i$ and $H_{3}=A_{2} A_{1}-$ $A_{0}>0$. The Routh-Hurwitz coefficient $H_{3}$ is given by

$$
H_{3}=(a+e+i)(a e+a i+c g+e i-f h)-a e i+a f h-c d h-c g e
$$

which simplifies to

$$
H_{3}=a(a e+a i+c g)+(e+i)(a e+a i+e i)+c g i-[c d+(e+i) f] h .
$$

Let $S_{3}$ be the region in the space of three by three matrices defined by the condition that all eigenvalues have negative real parts. At any point on the boundary of $S_{3}$ where the determinant is non-zero the eigenvalue configuration characteristic of the Hopf bifurcation must occur. In other words, two eigenvalues are imaginary and non-zero and the other is negative.

In the Michaelis-Menten system for the truncated MAPK cascade we have already exhibited a class of stationary solutions and shown that there is a choice of the parameters for which the matrix defined by the linearization of this system has negative determinant and the determinant of $M_{2}$ is negative. Call this point in parameter space $Z$. We recall that at $Z$, the constraints that have to be fulfilled are the following:

$$
\begin{array}{ll}
\frac{c_{2} d_{1}}{c_{1} d_{2}} & <1 \\
K K K_{\text {tot }} & =\sqrt{\frac{a_{2} a_{4}}{a_{1} a_{3}}} \\
q_{1} & =\sqrt{\frac{a_{1} a_{4}}{a_{2} a_{3}}}<1 \\
K K_{\text {tot }} & =\frac{q_{1}+2}{b_{1}\left(1-q_{1}\right)}+\delta,
\end{array}
$$

where $\delta>0$ is small. At $Z$ the linearization has negative trace. The coefficients will now be rescaled in a particular way. Let $\bar{a}_{i}$ be the values of the parameters $a_{i}$ at the starting point and define $a_{i}(L)=\bar{a}_{i} L$. The other parameters are kept fixed. Under this rescaling a stationary solution remains a stationary solution. The determinant of the linearization is rescaled by $L^{2}$ and so, in particular, has a fixed sign. The constants $d$ $i$ in the matrix of the linearization are scaled by a factor $L$ while the 
coefficients $a$ and $c$ are left unchanged. After rescaling the condition that the quantity $H_{3}$ is negative becomes

$L^{3}(f h-e i)(e+i)>L a(a e+a i+c g)+L^{2}(e+i)(a e+a i)+L^{2} c g i-L^{2} c d h$.

If we start the rescaling from $Z$ then for $L$ sufficiently large this condition will be satisfied. On the other hand, for $L$ sufficiently small and positive the opposite inequality will be satisfied. In other words, in this family the quantity $\mathrm{H}_{3}$ starts positive for $L$ small and positive and becomes negative at some large value of $L$. It must pass from being positive to being zero at some point $L_{0}$. In fact it must have a minimum for some negative value of $L$ and a maximum for some positive value of $L$ since it vanishes at the origin and its derivative there is positive. In fact the value $L_{0}$ is the unique positive root of a quadratic polynomial. At the unique positive value $L_{0}$ of $L$ for which it is zero this function has a negative derivative. It follows from the facts that $A_{2}>0$ and $A_{0}>0$ that $A_{1}$ cannot approach zero before $H_{3}$ reaches zero. Thus the family cannot leave $S_{3}$ before $H_{3}$ reaches zero. This family passes through a point where the eigenvalues satisfy the conditions for a Hopf bifurcation. When a three-dimensional matrix has one negative real eigenvalue $\mu_{1}$ and another eigenvalue $\mu_{2}$ which is not real the quantity $H_{3}$ is equal to

$$
-\operatorname{Re} \mu_{2}\left[\left(\operatorname{Re} \mu_{2}\right)^{2}+2\left(\operatorname{Im} \mu_{2}\right)^{2}+\left(\mu_{1}+\operatorname{Re} \mu_{2}\right)^{2}\right] .
$$

From this we can see that if a one-parameter family of matrices is such that the quantity $H_{3}$ passes through zero at some parameter value with nonzero velocity then the real part of the complex eigenvalue passes through zero at that point with non-zero derivative.

The situation which has just been described is as follows. We have a family of coefficients in the Michaelis-Menten system depending on the parameter $L$. There is a corresponding family of stationary solutions. We consider the linearization of the system at that point. We can suppose that for all values of $L$ considered it has one negative real eigenvalue $\mu_{1}$ and one eigenvalue $\mu_{2}$ which is never real. Moreover at $L=L_{0}$ the eigenvalue $\mu_{2}$ is imaginary and its real part moves through the imaginary axis with non-zero velocity as $L$ is varied through $L_{0}$. In other words, $\mu_{2}(L)$ defines a curve in the complex plane which passes through the imaginary axis transversely for $L=L_{0}$. In particular the linearization is always invertible. It was shown in 16 that the Michaelis-Menten system (35)(37) can be embedded into the MM-MA system (41)-(12) in such a way as to allow GSPT to be applied. In the terminology of [16] the transverse eigenvalues all have negative real parts. More information on these matters can be found in the appendix. Above we introduced a parameter $L$ into the MM system by letting the coefficients depend on $L$ in a certain way. This can be extended to the MM-MA system by making a smooth choice of corresponding coefficients for that system. After doing this the system on the slow manifold depends on the two parameters $\epsilon$ and $L$. At the point where the Hopf bifurcation occurs for $\epsilon=0$ and $L=L_{0}$ the derivative of the right hand side of the system with respect to the unknowns is invertible. Hence, by the implicit function theorem there is a unique nearby stationary solution depending smoothly on $L$ and $\epsilon$. Thus 
the family of stationary solutions for $\epsilon=0$ can be continued smoothly to a curve of stationary solutions in the slow manifold for small positive values of $\epsilon$. For each $\epsilon$ there are corresponding eigenvalues $\tilde{\mu}_{1}(L, \epsilon)=\mu_{1, \epsilon}(L)$ and $\tilde{\mu}_{2}(L, \epsilon)=\mu_{2, \epsilon}(L)$ of the linearization at the stationary solution. For $\epsilon$ small the curve $\mu_{2, \epsilon}$ intersects the imaginary axis transversely. Now the transverse eigenvalues are negative and it follows that the family of solutions of the MM-MA system undergoes a Hopf bifurcation. Using centre manifold theory the dynamics can be reduced to a two-dimensional situation for both the MM-MA system for the truncated Huang-Ferrell model and the corresponding MM system. Applying the theorem of Hopf in the form given in [15, Chapter VIII, Theorem 1.3 shows that in both cases there exists a one-parameter family of periodic solutions. The following theorem has been proved.

Theorem 1 There exist positive parameter values $a_{i}, b_{1}, c_{i}$ and $d_{i}$ such that the MM system for the truncated Huang-Ferrell model with these parameter values has a positive periodic solution. There exist positive parameter values $\tilde{a}_{i}, k_{i}$ and $\tilde{d}_{i}$ and positive values of the total amounts $E_{1, \text { tot }}$, $E_{2, \text { tot }},\left(K K P^{\prime} \text { ase }\right)_{\text {tot }}, K K K_{\text {tot }}$ and $K K_{\text {tot }}$ such that the $M M-M A$ system for the truncated Huang-Ferrell model with these parameter values and these values of the total amounts has a positive periodic solution.

\section{Bistability}

In 16] it was proved that there are parameter values for which the dual futile cycle has more than one stable stationary solution. It will now be shown that this property is inherited by the truncated MAPK cascade. For this the coefficients in the MM system for the truncated MAPK cascade will be rescaled. Define $c_{1}=\epsilon^{-1} \hat{c}_{1}, c_{2}=\epsilon^{-2} \hat{c}_{2}$ and $d_{2}=\epsilon^{-1} \hat{d}_{2}$. Then the equations for the quantities with hat are in the standard form for singular perturbation theory. For fixed values of $\overline{K K K}$ the equations for $[K K]$ and $[K K P P]$ are those for the dual futile cycle and we know that there are parameters for which there exist two stable hyperbolic stationary solutions. The evolution equation for $\overline{K K K}$ is

$$
\begin{aligned}
& \epsilon \frac{d}{d t}(\overline{K K K})=\frac{\hat{c}_{1}\left(K K K_{\mathrm{tot}}-\overline{K K K}\right)}{1+d_{1}\left(K K K_{\mathrm{tot}}-\overline{K K K}\right)} \\
& -\frac{\hat{c}_{2} \overline{K K K}}{\epsilon+\hat{d}_{2} \overline{K K K}+\epsilon b([K K]+[K K P])} .
\end{aligned}
$$

For $\epsilon=0$ this reduces to

$$
d_{1}\left(\frac{\hat{c}_{1} \hat{d}_{2}}{\hat{c}_{2} d_{1}}-1\right)\left(K K K_{\mathrm{tot}}-\overline{K K K}\right)=1 .
$$

Interestingly this last equation does not depend on $[K K]$ or $[K K P]$. Provided the quotient of reaction constants occurring in this equation, which is equal to $\frac{c_{1} d_{2}}{c_{2} d_{1}}$, is greater than one then a unique stationary solution is determined by solving this equation for $K K K_{\text {tot }}$ in terms of $\overline{K K K}$. It is 
an asymptotically stable and hyperbolic solution of the equation

$$
\frac{d}{d t}(\overline{K K K})=\frac{\hat{c}_{1}\left(K K K_{\mathrm{tot}}-\overline{K K K}\right)}{1+d_{1}\left(K K K_{\mathrm{tot}}-\overline{K K K}\right)}-\frac{\hat{c}_{2}}{\hat{d}_{2}} .
$$

Thus the transverse eigenvalue in the sense of GSPT is negative and that theory can be applied. It follows that the MM system for the truncated MAPK cascade exhibits bistability. This implies a corresponding statement for the MM-MA system. It can be shown in a similar way that both the MM and MM-MA systems contain saddle points for these values of the parameters.

It can also be shown that there are parameters for which there is a stationary value where the eigenvalues are real with the sign pattern $(-,-, 0)$. There $e i-f h<0$. Scaling with the parameter $L$ starting at this point gives a family of coefficients for which the determinant of the linearization is always zero. Along this family the quantity $H_{3}$ goes from positive to negative. When it crosses zero $A_{2}=0$ and there is a second zero eigenvalue. If the kernel of the matrix at that point were two-dimensional it would have to intersect the subspace spanned by the first two coordinates in a one-dimensional subspace. It would follow that this subspace was spanned by the vector with components $(0,1,0)$. This gives a contradiction. Thus the Jordan form of the matrix must be non-diagonalizable and the algebraic conditions on the linearization for a Bogdanov-Takens bifurcation are satisfied at that point. In other words, the condition BT.0 of [19] holds. Since, on the other hand, no information has been obtained on the genericity conditions BT.1-BT.3 this does not by itself give useful information on the dynamical properties of the solutions.

\section{The full Huang-Ferrell system}

The aim is now to reduce the full Huang-Ferrell system in a way similar to that done above for the truncated system. The first step is to introduce the quantity

$$
\overline{K K}=[K K P P]+[K \cdot K K P P]+[K P \cdot K K P P] .
$$

Consider the system of evolution equations for $\overline{K K K},[K K], \overline{K K},[K]$, $[K P P]$ and the substrate-enzyme complexes and rescale the unknowns. In this case the quantities not containing the $E_{i}, K K K, K K, K K P^{\prime}$ ase or $K P^{\prime}$ ase are not rescaled. These are the first three quantities in the fourth line of (2). Quantities which contain $K K$ or $K P^{\prime}$ ase but not $E_{i}, K K K$ or $K K P^{\prime}$ ase are rescaled by $\epsilon^{-1}$. These are the first three quantities in the second line, the last quantity in the fourth line and all quantities in the fifth line of (2). Quantities which contain $K K K$ or $K K P^{\prime}$ ase but not $E_{i}$ are rescaled by $\epsilon^{-2}$. These are the first two quantities in the first line, the last quantity in the second line and all quantities in the third line of (2). Quantities which contain the $E_{i}$ are rescaled by $\epsilon^{-3}$. These are the last four quantities in the first line of (2). The reaction constants $\tilde{a}_{1}$ and $\tilde{a}_{2}$ are multiplied by $\epsilon^{2}$ to get new quantities while $\tilde{a}_{3}, \tilde{a}_{4}, \tilde{a}_{5}$ and $\tilde{a}_{6}$ are multiplied by $\epsilon$. A new time coordinate is introduced as $\epsilon$ times the old 
one. These scalings have been chosen so that the new equations for the free substrates are independent of $\epsilon$ and the new equations for the substrateenzyme complexes have a factor $\epsilon$ in front of the time derivatives, just as in the case of the truncated system. The resulting equations extend (4)(12). The first two rescaled equations are unchanged and the third only differs from the corresponding equation for the truncated system in that $[K K P P]$ is replaced by $\overline{K K}$ on the left hand side. The list of expressions for the concentrations of the complexes can be extended as follows

$$
\begin{aligned}
& {[K \cdot K K P P]=\frac{\tilde{a}_{7}[K][K K P P]}{\tilde{d}_{7}+k_{7}},} \\
& {\left[K P \cdot K P^{\prime} \text { ase }\right]=\frac{\tilde{a}_{8}[K P]\left[K P^{\prime} \text { ase }\right]}{\tilde{d}_{8}+k_{8}},} \\
& {[K P \cdot K K P P]=\frac{\tilde{a}_{9}[K P][K K P P]}{\tilde{d}_{9}+k_{9}},} \\
& {\left[K P P \cdot K P^{\prime} \text { ase }\right]=\frac{\tilde{a}_{10}[K P P]\left[K P^{\prime} \text { ase }\right]}{\tilde{d}_{10}+k_{10}} .}
\end{aligned}
$$

There are the following additional equations for the total amounts of enzymes

$$
\begin{aligned}
& \overline{K K}=[K K P P]\left(1+K_{m, 7}^{-1}[K]+K_{m, 9}^{-1}[K P]\right), \\
& \left(K P^{\prime} \text { ase }\right)_{\text {tot }}=\left[K P^{\prime} \text { ase }\right]\left(1+K_{m, 8}^{-1}[K P]+K_{m, 10}^{-1}[K P P]\right) .
\end{aligned}
$$

The equations (29)- - 31 can be taken over to the full model except that in (31) the quantity $[K K P P]$ should be replaced by $\overline{K K}$ on the left hand side and that in order to obtain a closed system $[K K P P]$ must be substituted for in terms of $\overline{K K}$ on the right hand side. The following equations also hold:

$$
\begin{aligned}
& \frac{d}{d t}[K]=-\frac{k_{7}}{K_{m, 7}}[K][K K P P]+\frac{k_{8}}{K_{m, 8}}[K P]\left[K P^{\prime} \text { ase }\right] \\
& \frac{d}{d t}[K P P]=-\frac{k_{10}}{K_{m, 10}}[K P P]\left[K P^{\prime} \text { ase }\right]+\frac{k_{9}}{K_{m, 9}}[K P][K K P P] .(60)
\end{aligned}
$$

The evolution equations for the MM system are

$$
\begin{aligned}
& \frac{d}{d t}(\overline{K K K})=\frac{k_{1} K_{m, 1}^{-1} E_{1, \mathrm{tot}}[K K K]}{1+K_{m, 1}^{-1}[K K K]} \\
& -\frac{k_{2} K_{m, 2}^{-1} E_{2, \mathrm{tot}} \overline{K K K}}{1+K_{m, 2}^{-1} \overline{K K K}+K_{m, 3}^{-1}[K K]+K_{m, 5}^{-1}[K K P]} \\
& \frac{d}{d t}[K K]=-\frac{k_{3} K_{m, 3}^{-1} \overline{K K K}[K K]}{1+K_{m, 3}^{-1}[K K]+K_{m, 5}^{-1}[K K P]} \\
& +\frac{k_{4} K_{m, 4}^{-1}\left(K K P^{\prime} a s e\right)_{\mathrm{tot}}[K K P]}{1+K_{m, 4}^{-1}[K K P]+K_{m, 6}^{-1}[K K P P]} \\
& \frac{d}{d t}(\overline{K K})=+\frac{k_{5} K_{m, 5}^{-1} \overline{K K K}[K K P]}{1+K_{m, 3}^{-1}[K K]+K_{m, 5}^{-1}[K K P]}
\end{aligned}
$$




$$
\begin{aligned}
& -\frac{k_{6} K_{m, 6}^{-1}\left(K K P^{\prime} \text { ase }\right)_{\mathrm{tot}}[K K P P]}{1+K_{m, 4}^{-1}[K K P]+K_{m, 6}^{-1}[K K P P]}, \\
& \frac{d}{d t}[K]=-\frac{k_{7} K_{m, 7}^{-1} \overline{K K}[K]}{1+K_{m, 7}^{-1}[K]+K_{m, 9}^{-1}[K P]} \\
& +\frac{k_{8} K_{m, 8}^{-1}\left(K P^{\prime} \text { ase }\right)_{\mathrm{tot}}[K P]}{1+K_{m, 8}^{-1}[K P]+K_{m, 10}^{-1}[K P P]} \\
& \frac{d}{d t}[K P P]=+\frac{k_{9} K_{m, 9}^{-1} \overline{K K}[K P]}{1+K_{m, 7}^{-1}[K]+K_{m, 9}^{-1}[K P]} \\
& -\frac{k_{10} K_{m, 10}^{-1}\left(K P^{\prime} a s e\right)_{\mathrm{tot}}[K P P]}{1+K_{m, 8}^{-1}[K P]+K_{m, 10}^{-1}[K P P]}
\end{aligned}
$$

Assume that the coefficients $K_{m, i}^{-1}$ are equal to a common quantity $b_{2}$ for $7 \leq i \leq 10$. Extend the definition of $a_{i}$ by defining it to be $k_{i+2} K_{m, i+2}^{-1}$ for $i=5,7$ and $k_{i+2} K_{m, i+2}^{-1}\left(K P^{\prime} a s e\right)_{\text {tot }}$ for $i=6,8$. As in the case of the truncated system there exists a smooth choice of the parameters and total quantities of the enzymes for the MM-MA system which give rise to any choice of the parameters $a_{i}, b_{i}, c_{i}$ and $d_{i}$.

We now have a system describing the full cascade which is in the standard form of GSPT. To profit from this it is necessary to examine the transverse eigenvalues. These are the eigenvalues of the matrix which is the derivative of right hand side of the evolution equations for the enzyme-substrate complexes for fixed values of the substrate concentrations. There are ten complexes and this is a ten by ten matrix with components $L_{i j}$. Let the complexes be numbered in the order they are listed in (2). Each complex which does not share an enzyme contributes a diagonal element to the matrix. These are the components $L_{11}$ and $L_{22}$ and are negative. Each pair of complexes which share an enzyme contributes a two by two submatrix on the diagonal. They are the $L_{i j}$ with $2 k-1 \leq i, j \leq 2 k$ for $2 \leq k \leq 5$. The eigenvalues of each of these submatrices on the diagonal have negative real parts. The calculation is essentially the same as that done for the case of the truncated system in [16]. It remains to examine the effect of the non-zero elements of the matrix which do not belong to any of these blocks. These are $L_{23}, L_{24}, L_{67}$ and $L_{68}$. The elements $L_{11}$ and $L_{22}$ are alone in their columns. Thus the calculation of the eigenvalues reduces to that of the submatrix obtained by discarding the first and second rows and columns. Then the submatrices for $k=1$ and $k=4$ occur as direct sums with other matrices. Thus determining the eigenvalues reduces to doing so for the submatrix defined by $5 \leq i, j \leq 8$. This submatrix is block upper triangular and so its eigenvalues are the eigenvalues of the submatrices for $k=2$ and $k=3$. Combining these facts shows that all transverse eigenvalues have negative real parts.

Consider a stationary solution of the system (61)- 65) which satisfies $[K K]=[K K P P]$ and $[K]=[K P P]$. Explicit stationary solutions can be found in a way similar to what was done for the truncated system. It follows from equations (64) and (65) that $a_{5} \overline{K K}[K]=a_{6}[K P]$ and $a_{7} \overline{K K}[K P]=a_{8}[K P P]$. Hence $\overline{K K}^{2}=\frac{a_{6} a_{8}}{a_{5} a_{7}}$. The quantities $[K]$ and 
$[K P]$ are determined by

$$
[K]=\frac{K_{\mathrm{tot}}}{2+q_{2}}, \quad[K P]=\frac{q_{2} K_{\mathrm{tot}}}{2+q_{2}},
$$

where $q_{2}=\sqrt{\frac{a_{5} a_{8}}{a_{6} a_{7}}}$. The expression obtained for $\overline{K K K}$ in the case of the truncated system remains valid for the full system while in those for $[K K]$ and $[K K P]$ are modified to

$$
[K K]=\frac{K K_{\mathrm{tot}}}{2+\tilde{q}_{1}}, \quad[K K P]=\frac{q_{1} K K_{\mathrm{tot}}}{2+\tilde{q}_{1}}
$$

where $\tilde{q}_{1}=q_{1}+b_{2}\left(K_{\text {tot }}-[K]\right)$. In addition we have the relation

$$
K K_{\mathrm{tot}}=\frac{\left(2+\tilde{q}_{1}\right) \overline{K K}}{1+b_{2}\left(K_{\mathrm{tot}}-[K]\right)}
$$

so that $K K_{\text {tot }}$ is determined. Stationary solutions for the MM system for the full cascade can be determined as follows. Fix the parameters $a_{i}, b_{i}, c_{2}, d_{i}$. Then if $c_{1}$ is chosen sufficiently large the concentrations for the stationary solution can be expressed in terms of $K_{\text {tot }}$ and $K K_{\text {tot }}$. Note that if the parameters $a_{i}, 1 \leq i \leq 8$ are varied in such a way that $r_{k}=a_{2 k+1} / a_{2 k}$ remains unchanged for $1 \leq k \leq 4$ then $q_{1}$ and $q_{2}$ do not change and the stationary solution is preserved.

Expressing the evolution equations in terms of the quantities $a_{i}, b_{i}, c_{i}$ and $d_{i}$ and using the conservation laws gives

$$
\begin{aligned}
& \frac{d}{d t}(\overline{K K K})=\frac{c_{1}\left(K K K_{\mathrm{tot}}-\overline{K K K}\right)}{1+d_{1}\left(K K K_{\mathrm{tot}}-\overline{K K K}\right)} \\
& -\frac{c_{2} \overline{K K K}}{1+d_{2} \overline{K K K}+b_{1}\left(K K_{\mathrm{tot}}-\overline{K K}\right)} \\
& \frac{d}{d t}[K K]=-\frac{a_{1} \overline{K K K}[K K]}{1+b_{1}\left(K K_{\mathrm{tot}}-\overline{K K}\right)} \\
& \left.+\frac{a_{2}\left(K K_{\mathrm{tot}}-[K K]-\overline{K K}\right)}{1+b_{1}\left(K K_{\mathrm{tot}}-[K K]-\frac{b_{2}\left(K_{\mathrm{tot}}-[K P P]\right)}{1+b_{2}\left(K_{\mathrm{tot}}-[K P P]\right.}\right)} \overline{K K}\right) \\
& \frac{d}{d t}(\overline{K K})=\frac{a_{3} \overline{K K K}\left(K K_{\mathrm{tot}}-[K K]-\overline{K K}\right)}{1+b_{1}\left(K K_{\mathrm{tot}}-[K K]\right)} \\
& -\frac{a_{4} \overline{K K}}{b_{1} \overline{K K}+\left(1+b_{1}\left(K K_{\mathrm{tot}}-[K K]-\overline{K K}\right)\right)\left(1+b_{2}\left(K_{\mathrm{tot}}-[K P P]\right)\right)} \\
& \frac{d}{d t}[K]=-\frac{a_{5} \overline{K K}[K]}{1+b_{2}\left(K_{\mathrm{tot}}-[K P P]\right)} \\
& +\frac{a_{6}\left(K_{\mathrm{tot}}-[K]-[K P P]\right)}{1+b_{2}\left(K_{\mathrm{tot}}-[K]\right)}, \\
& \frac{d}{d t}[K P P]=+\frac{a_{7} \overline{K K}\left(K_{\mathrm{tot}}-[K]-[K P P]\right)}{1+b_{2}\left(K_{\mathrm{tot}}-[K P P]\right)} \\
& -\frac{a_{8}[K P P]}{1+b_{2}\left(K_{\mathrm{tot}}-[K]\right)}
\end{aligned}
$$


A relation will now be established between this system and the system for the truncated model by doing some rescaling. Replace $[K],[K P P]$ and $K_{\text {tot }}$ by $\epsilon[K], \epsilon[K P P]$ and $\epsilon K_{\text {tot }}$, respectively. Replace $a_{i}$ by $\epsilon^{-1} a_{i}$ for $5 \leq i \leq 8$. In the limit the first three equations are independent of $[K]$ and $[\overline{K P P}]$ and are just the equations of the truncated system analysed in the last section. The limit is in the form appropriate for applying GSPT. The linearization of the system for $[K]$ and $[K P P]$ is independent of those two variables. Its trace and determinant are $-\left(a_{5}+a_{7}\right) \overline{K K}-\left(a_{6}+a_{8}\right)<0$ and

$a_{5} a_{7} \overline{K K}^{2}+\left(a_{5}+a_{6}\right) a_{8}>0$, respectively and so the transverse eigenvalues have negative real parts. A parameter $L$ can be introduced in the system for the full cascade in the same way as was done for the truncated cascade. It follows that the presence of a Hopf bifurcation in the MM system for the truncated model implies that of Hopf bifurcation in the MM system for the full cascade. A parameter $L$ can also be introduced in the MM-MA system for the full cascade, implying the existence of a Hopf bifurcation for that system, i.e. the original system of Huang and Ferrell. Thus the following theorem has been proved.

Theorem 2 There exist positive parameter values $a_{i}, b_{i}, c_{i}$ and $d_{i}$ such that the MM system for the full Huang-Ferrell model with these parameter values has a positive periodic solution. There exist positive parameter values $\tilde{a}_{i}, k_{i}$ and $\tilde{d}_{i}$ and positive values of the total amounts $E_{1, \text { tot }}, E_{2, \text { tot }}$, $\left(K K P^{\prime} \text { ase }\right)_{\mathrm{tot}},\left(K K P^{\prime} \text { ase }\right)_{\mathrm{tot}}, K K K_{\mathrm{tot}}, K K_{\mathrm{tot}}$ and $K_{\mathrm{tot}}$ such that the $M M-M A$ system for the full Huang-Ferrell model with these parameter values and these values of the total amounts has a positive periodic solution.

\section{$6 \quad$ Further examples}

This section is concerned with some examples which are variations on those coming from the MAPK cascade. The first is a system which is similar to the truncated Huang-Ferrell system except that the second layer of the cascade only has one phosphorylation site. In other words, we discuss now the following cascade:

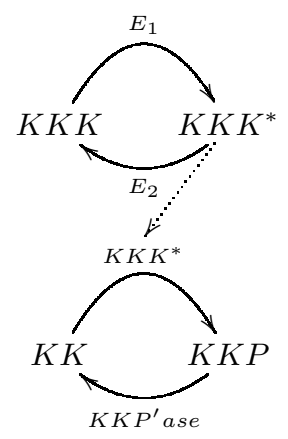

This minimal cascade and its generalization to several layers of simple phosphorylation loops have been considered in 30. where it is remarked that damped oscillations may occur in a system of this type. It will now 
be shown how the features observed for the MAPK cascade change in the case of a cascade of two simple phosphorylation loops. The variables are

$$
\begin{aligned}
& {[K K K],\left[K K K^{*}\right],\left[E_{1}\right],\left[E_{2}\right],\left[K K K \cdot E_{1}\right],\left[K K K^{*} \cdot E_{2}\right]} \\
& {[K K],[K K P],\left[K K P^{\prime} \text { ase }\right],\left[K K \cdot K K K^{*}\right],\left[K K P \cdot K K P^{\prime} \text { ase }\right](75)}
\end{aligned}
$$

We introduce the variable $\overline{K K K}=\left[K K K^{*}\right]+\left[K K \cdot K K K^{*}\right]$ in analogy to what was done in the previous examples. The variables are rescaled as in the truncated Huang-Ferrell model. This means that $[K K]$ and $[K K P]$ are not rescaled, for $[K K K],\left[K K K^{*}\right],\left[K K P^{\prime} a s e\right],\left[K K \cdot K K K^{*}\right]$ and $\left[K K P \cdot K K P^{\prime}\right.$ ase] the new quantity is $\epsilon^{-1}$ times the old one while for $\left[E_{1}\right],\left[E_{2}\right],\left[K K K \cdot E_{1}\right]$ and $\left[K K K^{*} \cdot E_{2}\right]$ the new quantity is $\epsilon^{-2}$ times the old one. The reaction constants $\tilde{a}_{1}$ and $\tilde{a}_{2}$ are multiplied by $\epsilon$ to get new quantities. A new time coordinate is introduced as $\epsilon$ times the old one. There results the following system

$$
\begin{aligned}
& \frac{d}{d t}(\overline{K K K})=-\tilde{a}_{2}\left[K K K^{*}\right]\left[E_{2}\right]+d_{2}\left[K K K^{*} \cdot E_{2}\right]+k_{1}\left[K K K \cdot E_{1}\right] \\
& \frac{d}{d t}[K K]=-\tilde{a}_{3}[K K]\left[K K K^{*}\right]+d_{3}\left[K K \cdot K K K^{*}\right]+k_{4}\left[K K P \cdot K K P^{\prime} \text { ase }\right](77) \\
& \epsilon \frac{d\left[K K K \cdot E_{1}\right]}{d t}=\tilde{a}_{1}[K K K]\left[E_{1}\right]-\left(d_{1}+k_{1}\right)\left[K K K \cdot E_{1}\right] \\
& \epsilon \frac{d\left[K K K^{*} \cdot E_{2}\right]}{d t}=\tilde{a}_{2}\left[K K K^{*}\right]\left[E_{2}\right]-\left(d_{2}+k_{2}\right)\left[K K K^{*} \cdot E_{2}\right] \\
& \epsilon \frac{d\left[K K \cdot K K K^{*}\right]}{d t}=\tilde{a}_{3}[K K]\left[K K K^{*}\right]-\left(d_{3}+k_{3}\right)\left[K K \cdot K K K^{*}\right] \\
& \left.\epsilon \frac{d\left[K K P \cdot K K P^{\prime} \text { ase }\right]}{d t}=\tilde{a}_{4}[K K P]\left[K K P^{\prime} \text { ase }\right]-\left(d_{4}+k_{4}\right)\left[K K P \cdot K K P^{\prime} \text { d(se }\right]\right)
\end{aligned}
$$

To get a closed system the following relations must be used.

$$
\begin{aligned}
& {\left[K K K^{*}\right]=\overline{K K K}-\left[K K \cdot K K K^{*}\right],} \\
& {[K K K]=K K K_{\mathrm{tot}}-\overline{K K K}+O(\epsilon),} \\
& {[K K P]=K K_{\mathrm{tot}}-[K K]+O(\epsilon),} \\
& {\left[E_{1}\right]=E_{1, \mathrm{tot}}-\left[K K K \cdot E_{1}\right],} \\
& {\left[E_{2}\right]=E_{2, \mathrm{tot}}-\left[K K K^{*} \cdot E_{2}\right],} \\
& {\left[K K P^{\prime} \text { ase }\right]=\left(K K P^{\prime} \text { ase }\right)_{\mathrm{tot}}-\left[K K P \cdot K K P^{\prime} \text { ase }\right] .}
\end{aligned}
$$

For $\epsilon=0$ the following MM system is obtained

$$
\begin{aligned}
& \frac{d}{d t}(\overline{K K K})=\frac{k_{1} K_{m, 1}^{-1} E_{1, \mathrm{tot}}[K K K]}{1+K_{m, 1}^{-1}[K K K]} \\
& -\frac{k_{2} K_{m, 2}^{-1} E_{2, \mathrm{tot}} \overline{K K K}}{1+K_{m, 2}^{-1} \overline{K K K}+K_{m, 3}^{-1}[K K]}, \\
& \frac{d}{d t}[K K]=-\frac{k_{3} K_{m, 3}^{-1} \overline{K K K}[K K]}{1+K_{m, 3}^{-1}[K K]} \\
& +\frac{k_{4} K_{m, 4}^{-1}\left(K K P^{\prime} \text { ase }\right)_{\mathrm{tot}}[K K P]}{1+K_{m, 4}^{-1}[K K P]} .
\end{aligned}
$$


The system (88)-(89) has the property that the trace of the derivative of the right hand side is always negative. Thus by the Dulac criterion this system admits no periodic solutions. It was shown in [8] that in this case the MM-MA system has a unique stationary solution for given values of the parameters and this means that the same is true for the MM system. On the boundary of the region of positive concentrations the vector field points inwards and all solutions are bounded due to the conservation laws. Putting all these facts together, it follows from Poincaré-Bendixson theory that the stationary solution of the MM system is globally asymptotically stable. It is not clear that stability might not be lost for general values of the parameters in the MM-MA system.

The next example is an in vitro model, introduced by Prabakaran, Gunawardena and Sontag [25], of the MAPK cascade consisting of the proteins Raf, MEK and ERK. The model system is simplified compared to the original biological system in two ways. The protein Raf is constitutively active. This corresponds to taking a fixed value of $\overline{K K K}$ in the model. ERK is mutated so that it can only be phosphorylated once, on tyrosine and not on threonine. The role of the phosphatase $K K P^{\prime}$ ase is played by PP2A (protein phosphatase $2 \mathrm{~A}$ ) and that of $K P^{\prime}$ ase by PTP (protein tyrosine phosphatase). Normally PP2A can remove a phosphate group from the threonine in ERK, thus causing a mixing of the layers but the mutation ensures that a phosphate of this kind is not present and PP2A cannot remove the phosphate from tyrosine. This leads to a cascade where the first layer allows two phosphorylation steps but the second only allows one. This cascade is represented in the following diagram:

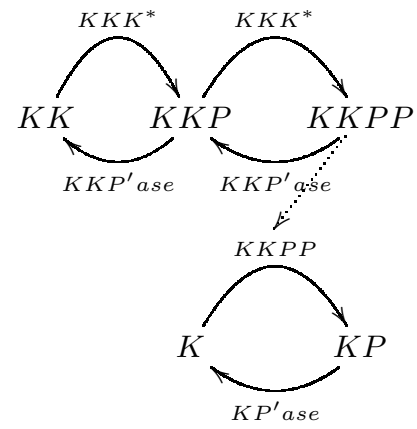

Suppose that the phosphorylation and dephosphorylation in the first layer are distributive and sequential. In other words, only one phosphate group is added or removed in each reaction, the groups are added in a specified order and removed in the reverse order. Given this data it is possible to set up an MM-MA model as done in other cases above but this is not the model used in 25]. There the phosphorylation of MEK is modelled using mass action (MA) kinetics with both phosphates being added in one step and the concentration of Raf not included as a variable. On the other hand the action of MEK as an enzyme in phosphorylating ERK is modelled in detail. This gives a kind of hybrid MA/MM-MA model, which we call the PGS model. It is proved in [25] that for the PGS model all solutions converge to a stationary solution at late times. 
Now the MM-MA model for the in vitro system of 25] will be examined, together with its MM reduction. In the Huang-Ferrell system discard the first four equations and take $\overline{K K K}$ to be a constant in the remaining equations. Then set $[K P \cdot K K P P],[K P P]$ and $\left[K P P \cdot K P^{\prime}\right.$ ase $]$ to zero together with the reaction constant $\tilde{a}_{9}$. Discard the last three equations. In the corresponding MM system this means taking $\overline{K K K}$ to be constant, setting $[K P P]$ and $a_{7}$ to zero and discarding the first and last equations. In this case the quantity $\overline{K K}$ is defined to be $[K K P P]+[K \cdot K K P P]$. It is no longer possible to keep the coefficients $K_{m, i}^{-1}$ with $7 \leq i \leq 10$ equal to the same positive constant $b_{2}$. This can be required for $i \neq 9$ but $K_{m, 9}^{-1}$ must be replaced by zero. This has the effect that the expression $b_{2}\left(K_{\text {tot }}-[K P P]\right)$ is replaced in equations (70), (71) and (72) by $b_{2}[K]$. The system of equations obtained is

$$
\begin{aligned}
& \frac{d}{d t}[K K]=-\frac{a_{1} \overline{K K K}[K K]}{1+b_{1}\left(K K_{\mathrm{tot}}-\overline{K K}\right)} \\
& +\frac{a_{2}\left(K K_{\mathrm{tot}}-[K K]-\overline{K K}\right)}{1+b_{1}\left(K K_{\mathrm{tot}}-[K K]-\frac{b_{2}[K]}{1+b_{2}[K]} \overline{K K}\right)}, \\
& \frac{d}{d t}(\overline{K K})=\frac{a_{3} \overline{K K K}\left(K K_{\mathrm{tot}}-[K K]-\overline{K K}\right)}{1+b_{1}\left(K K_{\mathrm{tot}}-[K K]\right)} \\
& -\frac{a_{4} \overline{K K}}{b_{1} \overline{K K}+\left(1+b_{1}\left(K K_{\mathrm{tot}}-[K K]-\overline{K K}\right)\right)\left(1+b_{2}[K]\right)} \\
& \frac{d}{d t}[K]=-\frac{a_{5} \overline{K K}[K]}{1+b_{2}[K]} \\
& +\frac{a_{6}\left(K_{\mathrm{tot}}-[K]\right)}{1+b_{2}\left(K_{\mathrm{tot}}-[K]\right)} .
\end{aligned}
$$

Next, in analogy with what has been done for other models above, stationary solutions will be considered which satisfy the restriction $[K K]=$ $[K K P P]$. These satisfy $a_{1}[K K] \overline{K K K}=a_{2}[K K P]$ and $a_{3}[K K P] \overline{K K K}=$ $a_{4}[K K]$. Hence $a_{1} a_{3} \overline{K K K}^{2}=a_{2} a_{4}$ and $\overline{K K K}=\sqrt{\frac{a_{2} a_{4}}{a_{1} a_{3}}}$. Substituting this back in gives $[K K P]=a_{1} a_{2}^{-1} \overline{K K K}[K K]=q_{1}[K K]$ where $q_{1}=\sqrt{\frac{a_{1} a_{4}}{a_{2} a_{3}}}$. Hence

$$
K K_{\text {tot }}=[K K]+[K K P]+\overline{K K}=\left(2+q_{1}+b_{2}[K]\right)[K K] .
$$

This allows $[K K]$ and $[K K P]$ to be expressed in terms of $K K_{\text {tot }}, q_{1}, b_{2}$ and $[K]$. These relations are equivalent to the first and second equations for stationary solutions. The remaining equation can be written as

$$
\overline{K K}=\frac{a_{6}\left(K_{\mathrm{tot}}-[K]\right)\left(1+b_{2}[K]\right)}{a_{5}[K]\left(1+b_{2}\left(K_{\mathrm{tot}}-[K]\right)\right)} .
$$

One way of determinining a set of stationary solutions is as follows. First choose $[K], K_{\text {tot }}>[K], b_{2}$, and the $a_{i}$. Then use the last equation to determine $\overline{K K}$. Next use

$$
K K_{\mathrm{tot}}=\frac{\left(2+q_{1}+b_{2}[K]\right) \overline{K K}}{1+b_{2}[K]} .
$$


Then $[K K],[K K P]$ and $[K K P P]$ are determined in such a way that all the equations for stationary solutions are satisfied. To summurize, we get equilibria for parameters, conserved quantities and concentrations parametrized over $[K], K_{\text {tot }}>[K], b_{2}$, and the $a_{i}$ by the following:

$$
\begin{aligned}
\overline{K K K} & =\sqrt{\frac{a_{2} a_{4}}{a_{1} a_{3}}} \\
K K_{\mathrm{tot}} & =\frac{a_{6}\left(K_{\mathrm{tot}}-[K]\right)\left(2+q_{1}+b_{2}[K]\right)}{a_{5}[K]\left(1+b_{2}\left(K_{\mathrm{tot}}-[K]\right)\right)} \\
{[K K] } & =\frac{a_{6}\left(K_{\mathrm{tot}}-[K]\right)}{a_{5}[K]\left(1+b_{2}\left(K_{\mathrm{tot}}-[K]\right)\right)} \\
\overline{K K} & =\frac{a_{6}\left(K_{\mathrm{tot}}-[K]\right)\left(1+b_{2}[K]\right)}{a_{5}[K]\left(1+b_{2}\left(K_{\mathrm{tot}}-[K]\right)\right)},
\end{aligned}
$$

where $q_{1}=\sqrt{\frac{a_{1} a_{4}}{a_{2} a_{3}}}$.

In order to prove the existence of a Hopf bifurcation it is tempting to proceed as in the analysis of the MM system for the truncated MAP kinase cascade. To do so we need to find parameters such that the coefficients $A_{0}, A_{1}$ and $A_{2}$ of the characteristic polynomial of the linearization are all positive. Then the parameters $a_{i}, i \in\{1,2,3,4\}$ should be rescaled with a positive constant $L$ so as to find a value $L_{0}$ for which the Hurwitz quantity $H_{3}=A_{1} A_{2}-A_{0}$ becomes zero. The linearization is very similar to that for the truncated MAP kinase cascade. Here again one entry is zero and all others have a sign. After a suitable change in the order of the variables only one sign differs. The resulting matrix of signs is

$$
\left[\begin{array}{lll}
- & 0 & - \\
+ & - & - \\
+ & - & -
\end{array}\right]
$$

The main problem we have encountered in trying to implement this strategy is to find a point in parameter space where the positivity of the $A_{i}$ holds. The coefficient $A_{0}$ is the negative of the determinant of the linearization. while the coefficient $A_{2}$ is the determinant of the analogue of the submatrix $M_{2}$ defined in the case of the truncated MAPK cascade (using the modified order of the variables). Hence the RouthHurwitz method will give us the desired factor $L_{0}$ only if the determinant of the linearization and that of $M_{2}$ have the same (negative) sign. Experiments with Maple indicate that these two determinants tend to have different signs for parameters satisfying the biologically motivated positivity conditions. The signs are governed by the signs of polynomials. When attempting to attain the relevant combination of signs by fixing some of the parameters and varying others the signs of the determinants are governed by those of two polynomials. In all experiments we did these polynomials were different but shared a unique positive zero where their signs changed. Unfortunately, having the sign of one of the determinants negative requires being on one side of this zero while having the other negative requires being on the other side of it. After trying many parameter configurations we are tempted to to conjecture that there is a deep reason why these determinants have systematically different signs. When 
we apply the Routh-Hurwitz method in the case that $A_{0}$ and $A_{2}$ have different signs it is still possible to find a unique positive value $L_{0}$ of $L$ with $H_{3}=0$. However instead of leading to the purely imaginary eigenvalues required for a Hopf bifurcation this leads to two real eigenvalues with equal magnitude and opposite sign.

\section{Conclusions and outlook}

The main result of this paper is a rigorous analytical proof of the existence of periodic solutions of the Huang-Ferrell system modelling the MAPK cascade. Their presence had been suggested by numerical and heuristic work in 26]. It was also proved that solutions of this type exist for cascades consisting of a layer with one phosphorylation followed by a layer with two phosphorylations but for the superficially similar case of a layer with two phosphorylations followed by a layer with one phosphorylation an attempt to obtain a similar proof ran into difficulties. The first of these two cases was considered in 26] but to the authors knowledge the second was not previously investigated in the literature. The methods used in the proofs are bifurcation theory and geometric singular perturbation theory. It should be noted that the heuristic considerations in [26], which might in principle have been used as a basis for proofs, were in the end hardly used at all. It would be interesting to know whether this alternative route could also be effective in this problem. Relevant ideas, involving relaxation oscillations and the Conley index, are explained in [2], 12] and [13.

An important question is to what extent the mathematical results obtained here apply to the real biological system. First it should be noted that in nature there is not just one MAPK cascade but many. The basic pattern is always the same but the details may be different. We now concentrate on the most famous example, the Raf-MEK-ERK cascade. This is essentially the example originally considered in 17. In that case Raf is replaced by Mos but the architecture of the system is identical. In this model it is assumed that the phosphorylations and dephosphorylations are distributive and sequential. Huang and Ferrell concentrate on this case but do mention that they also did simulations for the alternative versions where one or both of the kinases act in a distributive way. In 26, 35] and the bulk of the present paper only the distributive and sequential case is considered. It has been found that phosphorylation of ERK by MEK is distributive but not sequential [10, 3 while desphosphorylation of ERK has been found to be distributive and sequential 34. On the other hand the phosphorylation of MEK by Raf has been found to be processive [1]. See also [27] where it is remarked that the distinction between the two mechanisms may not be absolute - processive phosphorylation may be thought of as a limiting case of distributive phosphorylation where the second step takes place much faster than the first. This means that the original Huang-Ferrell model is not applicable to the Raf-MEK-ERK cascade. It is also known that there are cases in which processive phosphorylation suppresses complicated dynamical behaviour (in this case bistability) present when the phosphorylation is distributive [4], 5]. Thus it would be interesting to investigate whether there are os- 
cillations in the system obtained by modifying the Huang-Ferrell model by making the phosphorylation in the second layer processive.

It is also interesting to know what effect further interactions between proteins not included in the Huang-Ferrell model might have on the dynamics. It was observed in 20] that binding of Raf to MEK can influence the dynamics of the MAPK cascade, enhancing bistability. In real biological systems the MAPK cascade is also embedded in various external feedback loops. One well-known example is that ERK has a suppressive effect on Raf via the guanine nucleotide exchange factor son of sevenless (SOS). That the resulting negative feedback could lead to oscillations was observed theoretically in [18. Sustained oscillations in the MAPK cascade have been observed experimentally in 28. They have a period of about 15 minutes and have been observed to continue for over ten hours. A quantitative comparison with simulations indicates that these oscillations are not due to sequestration effects intrinsic to the cascade but to the feedback loop via SOS. Another type of feedback leading to oscillations which involves sequestration but is not intrinsic to the cascade is discussed in 21. In that case the binding of activated ERK to a substrate reduces its availability for dephosphorylation. These examples make it clear that there are numerous examples of biological interest which represent potential applications of the methods developed in the present paper.

Another key issue is that of the biological role of complicated dynamical features such as bistability, sustained oscillations or chaos in the MAPK cascade, with or without external feedback. Many different signals pass through this cascade and it may be that non-trivial dynamics can be used to encode information, for instance by frequency modulation of oscillations. Here it could be useful to compare with other biological systems where this type of phenomenon is believed to be important, such as the $\mathrm{NF} \kappa \mathrm{B}$ pathway 29 or calcium signalling 7 . On the other hand it could be that complicated dynamical behaviour in the basic MAPK cascade is an unwanted side effect and that the feedback loops in which it is embedded in biological systems serve to suppress it. Further mathematical investigations of systems related to the MAPK cascade could serve to understand the cascade itself better in its biological context and could also produce new insights into the architecture of biochemical systems. It should also be kept in mind that a better understanding of the dynamics of the cascade could be important for medical progress [31. In chemotherapy of cancer Raf inhibitors are already in use while MEK inhibitors have been the subject of extensive clinical trials but have not yet been effective. A better theory of the system could help to understand where to look for appropriate drugs.

One question which has been left open here is that of the stability of the periodic solutions whose existence has been proved. Is it possible to develop methods to prove that in some of these models there are parameters for which the first Lyapunov coefficient is non-zero, which would solve the stability problem? Is it possible to extend the techniques used here to prove the existence of fold-Hopf or Hopf-Hopf bifurcations in the Huang-Ferrell model and to check the associated genericity conditions which would give information on chaotic behaviour? Evidently, modelling the MAPK cascade gives rise to a large number of challenging 
mathematical problems.

\section{Appendix: geometric singular perturba- tion theory (GSPT)}

In this appendix some results concerning GSPT needed in the paper will be collected. In [16] a theorem from [9] was applied but in this paper we need a parameter-dependent version of that result which does not obviously follow from the theorem. In fact, starting from the basic transformations carried out in 9] and the idea of a slow manifold, the statements we need can be proved using standard results from the theory of centre manifolds. This will now be explained.

The starting point is a system of equations of the form

$$
\begin{aligned}
& \dot{x}=f(x, y, \alpha, \epsilon), \\
& \epsilon \dot{y}=g(x, y, \alpha, \epsilon),
\end{aligned}
$$

where $x, y$ and $\alpha$ belong to open neighbourhoods of the origin in $\mathbb{R}^{n_{1}}$, $\mathbb{R}^{n_{2}}$ and $\mathbb{R}^{k}$ respectively and $\epsilon$ belongs to an interval of the form $\left[0, \epsilon_{0}\right)$. The dot stands for $d / d t$. It will be assumed that the functions $f$ and $g$ are smooth and that they can be extended to smooth functions in a neighbourhood of $\epsilon=0$. This system is now transformed as in [9] by defining a new time coordinate by $\tau=t / \epsilon$ and treating $\epsilon$ and $\alpha$ as new unknowns. The result is

$$
\begin{aligned}
x^{\prime} & =\epsilon f(x, y, \alpha, \epsilon), \\
y^{\prime} & =g(x, y, \alpha, \epsilon), \\
\alpha^{\prime} & =0, \\
\epsilon^{\prime} & =0,
\end{aligned}
$$

where the prime denotes $d / d \tau$. Observe now that the solutions of the equation $g(x, y, \alpha, 0)=0$ are stationary solutions of the system (104)-(107). We assume that $g(0,0,0,0)=0$. Suppose that there exists a smooth function $h_{0}$ such that $g(x, y, \alpha, 0)=0$ is equivalent to $y=h_{0}(x, \alpha)$. The centre subspace of the stationary point at the origin is of dimension at least $n_{1}+k+1$. We now ensure that its dimension is no greater than that by assuming that all eigenvalues of the linearization of the system at the origin other than the zero eigenvalues arising from the manifold of stationary solutions already mentioned and that coming from equation (107) have non-zero real parts. These will be called the transverse eigenvalues. They are the eigenvalues of $D_{y} g(0,0,0,0)$. For any positive integer $l$ the centre manifold theorem ([19], Theorem 5.1) implies that there exists a centre manifold $M_{c}$ of the origin of class $C^{l}$ and of dimension $n_{1}+k+1$. Another well-known result about centre manifolds states that any stationary solutions sufficiently close to a given stationary solution must lie on its centre manifold. Thus the solutions of $g(x, y, \alpha, 0)=0$ all lie on the centre manifold of the origin. Since the dimension of the centre subspace is the 
same for all of these points it follows that $M_{c}$ is also a centre manifold of these neighbouring points. $M_{c}$ is what is called the slow manifold. In a neighbourhood of the origin it can be written in the form $y=h(x, \alpha, \epsilon)$ for a $C^{l}$ function $h$ with $h(x, \alpha, 0)=h_{0}(x, \alpha)$. Considering the restrictions of the dynamical system with the intersections of $M_{c}$ with the subspaces of constant $\alpha$ and $\epsilon$ gives rise to a dynamical system depending in a regular way on the parameters $\alpha$ and $\epsilon$. Its explicit form (when written in terms of the time coordinate $t$ ) is

$$
\dot{x}=f(x, h(x, \alpha, \epsilon), \alpha, \epsilon) .
$$

In this way the singular limit $\epsilon \rightarrow 0$ in the original system has been reduced to a regular limit. For $\epsilon=0$ it reduces to

$$
\dot{x}=f\left(x, h_{0}(x, \alpha), \alpha, 0\right)
$$

\section{References}

[1] Alessi, D. R., Saito, Y., Campbell, D. G., Cohen, P., Sithanandam, G., Rapp, U., Ashworth, A., Marshall, C. J. and Cowley, S. 1994 Identification of the sites in MAP kinase kinase-1 phosphorylated by p74raf-1. EMBO J. 13, 1610-1619.

[2] Angeli, D., Ferrell, J.E. Jr. and Sontag, E. D. 2004 Detection of multistability, bifurcations and hysteresis in a large class of biological positive-feedback systems. Proc. Natl. Acad. Sci. USA 101, 18221827.

[3] Burack, W. R. and Sturgill, T. W. 1997 The activating dual phosphorylation of MAPK by MEK is nonprocessive. Biochemistry 36, 5929-5933.

[4] Conradi, C., Saez-Rodriguez, J., Gilles, E.-D. and Raisch, J. 2005 Using chemical reaction network theory to discard a kinetic mechanism hypothesis. IEE Proc. Systems Biology 152: 243-248.

[5] Conradi, C. and Shiu, A. 2015 A global convergence result for processive multisite phosphorylation systems. Bull. Math. Biol. 77, 126-155.

[6] Del Vecchio, D., Ninfa, A. J. and Sontag, E. D. 2008 Modular cell biology: retroactivity and insulation. Nature Mol. Syst. Biol. 4, 161.

[7] Falcke, M. 2004 Reading the patterns in living cells - the physics of $\mathrm{Ca}^{2+}$ signaling. Adv. Phys. 53, 255-440.

[8] Feliu, E. and Wiuf, C. 2012 Enzyme-sharing as a cause of multistationarity in signalling systems. J. R. Soc. Interface 9, 1224-1232.

[9] Fenichel, N. 1979 Geometric singular perturbation theory for ordinary differential equations. J. Diff. Eq. 31, 53-98.

[10] Ferrell, J. E., Jr. and Bhatt, R. R. 1997 Mechanistic studies of the dual phosphorylation of mitogen-activated protein kinase. J. Biol. Chem. 272, 19008-19016.

[11] Gantmacher, F. R. 1959 The theory of matrices, Vol. II, Chelsea, New York. 
[12] Gedeon, T. and Sontag, E. D. 2007 Oscillations in multi-stable monotone systems with slowly varying feedback. J. Diff. Eq. 239, 273-295.

[13] Gedeon, T. 2010 Oscillations in monotone systems with a negative feedback. SIAM J. Dyn. Sys. 9, 84-112.

[14] Grimbs, S., Arnold, A., Koseska, A., Kurths, J., Selbig, J. and Nikoloski, Z. 2011 Spatiotemporal dynamics of the Calvin cycle: multistationarity and symmetry breaking instabilities. Biosystems 103, 212-223.

[15] Hale, J. K. 1969 Ordinary Differential Equations. Dover, Mineola.

[16] Hell, J. and Rendall, A. D. 2015 A proof of bistability for the dual futile cycle. Nonlin. Anal. RWA 24, 175-189.

[17] Huang, C.-Y. F. and Ferrell, J. E., Jr. 1996 Ultrasensitivity in the mitogen-activated protein kinase cascade. Proc. Natl. Acad. Sci. USA 93, 10078-10083.

[18] Kholodenko, B. N. 2000 Negative feedback and ultrasensitivity can bring about oscillations in the mitogen-activated protein kinase cascades. Eur. J. Biochem, 267, 1583-1588.

[19] Kuznetsov, Y. A. 2010 Elements of applied bifurcation theory. Springer, Berlin.

[20] Legewie, S., Schoeberl, B., Bluthgen, N, and Herzel, H. 2007 Competing docking interactions can bring about bistability in the MAPK cascade. Biophys. J. 93, 2279-2288.

[21] Liu, P., Kevrekidis, I. G. and Shvartsman, S. V. 2011 Substratedependent control of ERK phosphorylation can lead to oscillations. Biophys. J. 101, 2572-2581.

[22] Markevich, N. I., Hoek, J. B. and Kholodenko, B. N. 2004 Signaling switches and bistability arising from multisite phosphorylation in protein kinase cascades. J. Cell Biol. 164, 353-359.

[23] Murray, J. D. 1989 Mathematical biology. Springer, Berlin.

[24] Ortega, F., Garcés, J. L., Mas, F., Kholodenko, B. N. and Cascante, M. 2006 Bistability from double phosphorylation in signal transduction. FEBS J. 273, 3915-3926.

[25] Prabakaran, S., Gunawardena, J. and Sontag, E. D. 2014 Paradoxical results in perturbation-based network reconstruction. Biophys. J. 106, 2720-2728.

[26] Qiao, L., Nachbar, R. B., Kevrekidis, I. G. and Shvartsman, S. Y. 2007 Bistability and oscillations in the Huang-Ferrell model of MAPK signalling. PLoS Comp. Biol. 3, 1819-1826.

[27] Schilling, M., Maiwald, T., Hengl, S., Winter, D., Kreutz, C, Kolch, W., Lehmann, W. D., Timmer, J. and Klingmüller, U. 2009 Theoretical and experimental analysis links isoform specific ERK signalling to cell fate decisions. Mol. Sys. Biol. 5, 334.

[28] Shankaran, H., Ippolito, D. L., Chrisler, W. B., Resat, H., Bollinger, N., Opresko, L. K. and Wiley, H. S. 2009 Rapid and sustained nuclear-cytoplasmic ERK oscillations induced by epidermal growth factor. Mol. Sys. Biol. 5, 332. 
[29] Sung, M.-H., Salvatore, L., De Lorenzi, R., Indrawan, A., Pasparakis, M., Hager, G. L., Bianchi, M. E. and Agresti, A. 2009 Sustained oscillations of $\mathrm{NF} \kappa \mathrm{B}$ produce distinct genome scanning and gene expression profiles. PloS ONE 4(9):e7163.

[30] Ventura, A. C., Sepulchre, J.-A. Merajver, S. D. 2008 A hidden feedback in signalling cascades is revealed. PLoS Comp. Biol. 4(3):e1000041.

[31] Ventura, A. C., Jackson, T. L. and Merajver, S. D. 2009 On the role of cell signalling models in cancer research. Cancer Res. 69, 400-402.

[32] Ventura, A. C. and Sepulchre, J.-A. 2013 Intrinsic feedbacks in MAPK signalling cascades lead to bistability and oscillations. Acta Biotheor. 61, 59-78.

[33] Wang, L. and Sontag, E. D. 2008 On the number of steady states in a multiple futile cycle. J. Math. Biol. 57, 29-52.

[34] Zhao, Y. and Zhang, Z. Y. 2001 The mechanism of dephosphorylation of extracellular signal-regulated kinase 2 by mitogen-activated protein kinase phosphatase 3. J. Biol. Chem. 276, 32382-32391.

[35] Zumsande, M. and Gross, T. 2010 Bifurcations and chaos in the MAPK signalling cascade. J. Theor. Biol. 265, 481-491. 